\title{
Lambertian Luminous Intensity Radiation Pattern Analysis in OLOS Indoor Propagation for Better Connectivity
}

\author{
Vaishali, ${ }^{1}$ Sandeep Sancheti, ${ }^{2}$ Arvind Dhaka $\left({ }^{D},{ }^{3}\right.$ Amita Nandal, ${ }^{3}$ \\ Hamurabi Gamboa Rosales $\mathbb{D}^{\mathbb{D}},{ }^{4}$ Deepika Koundal, ${ }^{5}$ Francisco Eneldo López Monteagudo, ${ }^{4}$ \\ Carlos Eric Galvan Tajada, ${ }^{4}$ and Arpit Kumar Sharma ${ }^{3}$
}

\author{
${ }^{1}$ Tishitu Technology \& Research Private Limited, Jaipur, Rajasthan 302017, India \\ ${ }^{2}$ Department of Information \& Communication Technology, Marwadi University, Rajkot, Gujarat 360003, India \\ ${ }^{3}$ Department of Computer and Communication Engineering, Manipal University Jaipur, Jaipur, India \\ ${ }^{4}$ Unidad Academica de Ingenieria Electrica, Universidad Autonoma de Zacatacas, Jardin Juarez 147, Centro Historico, \\ ZA 98000, Mexico \\ ${ }^{5}$ School of Computer Science, University of Petroleum \& Energy Studies, Dehradun, India
}

\begin{abstract}
Correspondence should be addressed to Arvind Dhaka; arvind.neomatrix@gmail.com and Hamurabi Gamboa Rosales; hamurabigr@uaz.edu.mx
\end{abstract}

Received 11 October 2021; Accepted 17 January 2022; Published 22 February 2022

Academic Editor: Carles Gomez

Copyright (C) 2022 Vaishali et al. This is an open access article distributed under the Creative Commons Attribution License, which permits unrestricted use, distribution, and reproduction in any medium, provided the original work is properly cited.

\begin{abstract}
This paper illustrates the impact of wavelength on the Optical Line of sight (OLOS) link working in the Visible Light Communication (VLC) spectrum. The extension of work is based on previous outcomes given by researchers. It has been elaborated with different determinants based on the color factor of LED available in the Visible Light (VL) spectrum. In the modern world, Light Emitting Diodes (LEDs) are prevailing the market as a light source, which is dominantly used at homes and workplaces. The designed VLC system includes LED as a transmitter, which is used as a higher source of brightness and provides both illuminations and data communication. Solid-state lighting characteristic shows the electroluminance capability of high brightness LEDs. Within few years, the luminous efficacy of LED is exponentially increasing from the range of less than $0.1 \mathrm{~lm} / \mathrm{W}$ to over $230 \mathrm{~lm} / \mathrm{W}$ with a huge lifetime of around 100,000 h. MATLAB is used to simulate a VLC link between LEDs (operated at different wavelengths) and photodetector. We present here the performance analysis of Lambertian Luminous Intensity radiation pattern (LLIRP) generated by different colors of LED for a clear OLOS model with different Spectral Luminous Efficiency at photonic vision. A designed system model of VLC supports short links present in indoor room conditions with better connectivity.
\end{abstract}

\section{Introduction}

Optical wireless communication (OWC) has become a trending field among researchers because of the high capacity that it offers to solve last-mile applications $[1,2]$. Visible light communication arises as to the latest research trend in indoor optical wireless communication (OWC), which contributes the most matured and robust solutions to many issues. Nowadays, VLC is not only limited to being a source of light in home networking but also provides highspeed communication networks via light links in offices, airplanes, traffic light signals, etc. $[3,4]$. In the last few years, growing research in VLC shows the reliability and power efficiency offered by LED, which is to date much more remarkable than any conventional incandescent source of light used for lighting. Recent research in VLC demonstrates successfully data transmission up to 3 Gbps over short links installed in indoor environments [5]. The main drivers of this technique include high bandwidth/data rate, longer lifetime LEDs as compared with incandescent bulbs, better brightness level, no health and physical hazards, data security, and low power consumption $[6,7]$. Due to such advantages, LEDs show the potential for higher data communication with power-saving features on global platforms. 
The main problem of indoor VLC links is only a requirement of precise alignment and pointing with perfect directionality of the source of light with the receiver.

The infrastructure of any commercial organization and office buildings offer light wave as optical light emitting diodes (OLED), which has a tremendous potential to deploy the several access points (AP) for light fidelity (Li-Fi) [8]. LiFi technology is also notable to many as an interference-free system with other existing technologies (e.g., Wi-Fi, Ethernet, WiMAX, etc.) due to the use of the terahertz spectrum. Rapid growth in multimedia communication needs a smart indoor wireless network for better connectivity with the number of portable appliances. Such technologies can be easily installed by just using inexpensive optoelectronic devices like OLED connected with silicon photodetectors [9]. Since the last decade, LEDs have been considered a general source of illumination only, but now it becomes an alternative source of high-speed communication. The first visible-light LEDs operated only at low intensity and were limited to wavelength of red color. Nevertheless, modern LEDs are available in vast ranges of visible, ultraviolet, and infrared wavelengths, with very high illuminance and low power consumption. As compared with other sources of illumination like traditional incandescent, luminous efficacy is limited to $52 \mathrm{~lm} / \mathrm{W}$ only. While in the case of fluorescent lamps, it is limited to $90 \mathrm{~lm} / \mathrm{W}$ [10]. Although, LEDs show their peak efficiency over $260 \mathrm{~lm} / \mathrm{W}$, which is much lower when compared with its theoretically predicted value that reaches up to $425 \mathrm{~lm} / \mathrm{W}$ [11]. Coming years make us the witness of about rapid increase in performance level offered by illumination of OLEDs.

In recent times, visible light communication (VLC) has gained huge consideration from the academic community and industry. Indeed, the spectrum crunch of $\mathrm{Wi}-\mathrm{Fi}$ is seeking the alternative of radiofrequency communication, and VLC becomes the front runner due to its simplicity, speed, full-duplex nature, license-free operation, and data security. Furthermore, VLC achieves several landmarks by offering new applications such as underwater communication, vehicle-to-vehicle communication, and indoor localization. VLC system plays a key role by selecting the accurate transmitter (TX) and receiver (RX) to attain and sustain high performance. The high performance majorly depends on the performance of the source, i.e., LEDs. The modulation needs a perfect source that can provide both the illumination and the communication channel. Numerous aspects such as the LED type, color, illumination power, and bandwidth can put a direct influence on performance. Therefore, the selection of exact LED is quite significant according to the VLC scenarios, particularly where the LEDs need to perform the bidirectional communication.

The bidirectional performance between LEDs can be affected by several factors and environmental circumstances. For example, the illumination of LED alters according to field-of-view and distance. The ambiance of light can also vary the LED communication and its performance. In brief, the selection of receiving mechanism is also critical due to the high sensitivity of photodiodes (PDs) for low-power LEDs. This paper elaborates the examination on accurate usage of LEDs in VLC systems as tans-receiver. This work presents the evaluation of the LED impact on the VLC performance by varying the color under realistic environments. It becomes significant to understand the performance of LED (based on color), as it helps to understand the power losses with the change of distance. Our results validate that the color intensity of LED can greatly influence performance and communication quality. For instance, the green LED utilization can provide a high luminous intensity for the yellow, blue, and red LEDs. Such LEDs are very common in use and easily available. It is important to consider the fact that we need to work on such sources that are easily available. While prior research studies only target to assess physical properties of visible light or only work on a single parameter, our work opens new perspectives by considering the power losses. Different angles approach optical gain, modulation schemes, multiple access, and applications.

The proposed system offers several benefits as this model accurately predicts the intensity pattern of the source towards the receiver. LLIRP can estimate the received power distribution according to any room dimension. It helps in avoiding propagation losses and provides optimum efficiency. Furthermore, the mathematical derivations provide the precise calculation of the luminous intensity index based on radiant intensity and luminous intensity pattern. Such mathematical expressions can derive the received power of the room at any point. Further investigation is made on the calculation of transmitting LEDs' radiation pattern on the performance of received power. It becomes significant to measure the impact because it can help in transferring data at high speed. The proposed model has the potential to evaluate the performance of the receiver based on field of view and half power angle. Coordination between transmitter and receiver is important in order to develop an effective VLC system. The LLIRP model not only describes the characteristics of the source (wavelength dependent) but also focuses on the receiver's performance.

This paper deals with extensive work performed on the distribution of luminous intensity (LI) of light under various assumptions to set an optical line of sight link for VLC communication. The distribution of LIs has been analyzed in the form of Lambertian radiation pattern (LRP) that shows a clear variation in results when operated on different wavelengths in the visible spectrum. A radiation pattern describes the relative strength of light emitted in any direction from the light source, which is further related to Lambert's cosine law [12]. Based on practical results achieved as LLIRP on MATLAB simulations, additional work has been performed on the influence of LRP on the received power of photodetector.

\section{Previous Work}

In previous literature, researchers studied the color impact of LED on VLC in different manners. The primary methodologies focus on obtaining the correlation among several modulation schemes with different colors. For instance, IEEE 802.15.7.5 depicts the Color-Shift Keying approach. Furthermore, researchers proposed some other color-based 
modulation mechanisms after the formation of this standard, such as generalized color modulation (GCM). This work has substantially used a different approach as several prior works utilized photodiodes (PDs) or cameras as receivers. At the same time, the present study focuses on the characteristics of LEDs that make them efficient transreceiver for bidirectional communication. Full duplex communication among LEDs is conceivable as LEDs can work like photo sensors that have the potential to receive light even beyond the emitted frequency. Several studies have worked on defining and assessing the characteristics of LEDs. It starts with the assessment of the emission/reception spectrum of numerous LEDs based on their colors with their significant chemical applications as absorption sensors. Kowalczyk et al. suggested significant VLC application, however, restricted only in the selection of amber/red LEDs as transreceivers [13].

Since 2003, numerous research works have been performed on the efficient bidirectional utilization of LEDs under VLC conditions. Dietz et al. proposed the first experimental study that considered the utilization of LEDs as the transreceivers for the full-duplex channel [14]. Giustiniano et al. have further designed the VLC system with low complexity considering the full-duplex transmission of data between LEDs [15]. The study depicted the primary concept of the different LED's impact on a VLC interface by conducting several experimentations under two scenarios, such as blue-to-blue and red-to-red LEDs to compare and attain superior performance. Afterward, Schmid et al. suggested another work on a full-duplex interface among LEDs and assessed the link performance by considering several aspects like energy consumption and communication range [16]. However, the outcomes were advanced to the prior studies as the link distances are greater than $2 \mathrm{~m}$ and the data transmission rate is $800 \mathrm{~b} / \mathrm{s}$. Further, the model operates only on the same color LEDs. Wang et al. used PDs/LEDs as the receivers by introducing a research platform as OpenVLC for full-duplex transmission [17]. This work only considers red LEDs for data transmission and made their evaluations. Although, our work provides the insights of several colors with their respective luminous intensities and their impact on data transmission in terms of power losses, this work is helping to gain better approaches while developing the fullduplex transmission by using LEDs.

VLC systems prove their worth by providing new perspectives for bidirectional communication using LEDs with their characteristics as highly efficient photosensors. For instance, Li et al. designed a novel model as a MIMO-VLC system utilizing LEDs. Such transreceivers worked on RGB wavelength and outlining the emission/reception potentials of LEDs while creating a system that has the potential to perform multiple communications [18]. The experts assessed the performance of data transmission regarding several color combinations. The authors concluded that blue-green and red-red interfaces do not experience interferences from each other.

Bhalerao et al. suggested a line of site model by using Lambertian pattern of LED in which they analyzed power distribution of white LED source with a photodiode as a receiver in a room. This investigation completely depends upon the half-power angle of the LED source with the field of view of photodetector and mode number of radiation lobe [19]. Wang et al. developed a new theory for the analysis of received power distribution in a generalized indoor VLC system. The goal of their system is to develop a theory related to the location of LED, in which mathematical expressions of received power are derived for both wall-mounted and ceiling-mounted LED source layouts. Their model also explains the dependency of received power with illumination [20].

Chien et al. propose a novel and accurate analytical representation of radiation patterns, generated by an LED. Two major constraints as angular intensity distribution and the irradiance spatial pattern are taken into account during their analysis. A mathematical model is demonstrated, tested, and matched with datasheets of LEDs belonging to several major manufacturers [21]. In another work, Moreno explains about Spatial distribution of LED radiation. This model gives an analytical relationship between radiated patterns and the main constraints of LEDs, which are chip location, chip shape, chip radiance, encapsulant refractive index, encapsulant geometry, and cup reflector [22]. Our paper is the extended version of previous work in the form of analyzing the impact of change in wavelengths of light on Lambertian Luminous Intensity radiation pattern. There are some connections present between their work and the problems that encourage us to analyze the following issues in-depth but with different objectives.

This paper presents the expansion of several concepts considering the subsequent aspects as performing the work involving the LEDs diversity on a broader scale to widely explore the visible spectrum based on each color (red, green, yellow, and blue). Furthermore, this study illustrates the detailed experimental analysis by considering the most common colors (RGB) for full-duplex data transmission with the help of the Lambertian luminous intensity model, which is an accurate mathematical analysis with their respective simulation results. This work presents all the experimental assessments according to real-world VLC scenarios. In conclusion, this research study opens space for deliberations associated with recognizing the future aspects of VLC systems, e.g., medium access mechanisms and channel allocation depending on LED colors.

\section{VLC System Model}

A Clear Line of Sight model based on the light wave is designed under a free-space optical channel for indoor room dimensions, as shown in Figure 1. This design is applicable with some assumptions that all LED sources are placed at the center of the room and on the ceiling, i.e., at the top level, but in practicality, it may be possible that the position of source places is at horizontal plane like nearby windows. Another one is that there is a uniform distribution of luminance over the small active area of the receiver at receiving plane. Consideration of this assumption is due to the fact of using a solar panel as a photodetector (PD) in many VLC systems that consist of an active area of several hundred $\mathrm{cm}^{2}$. The last 


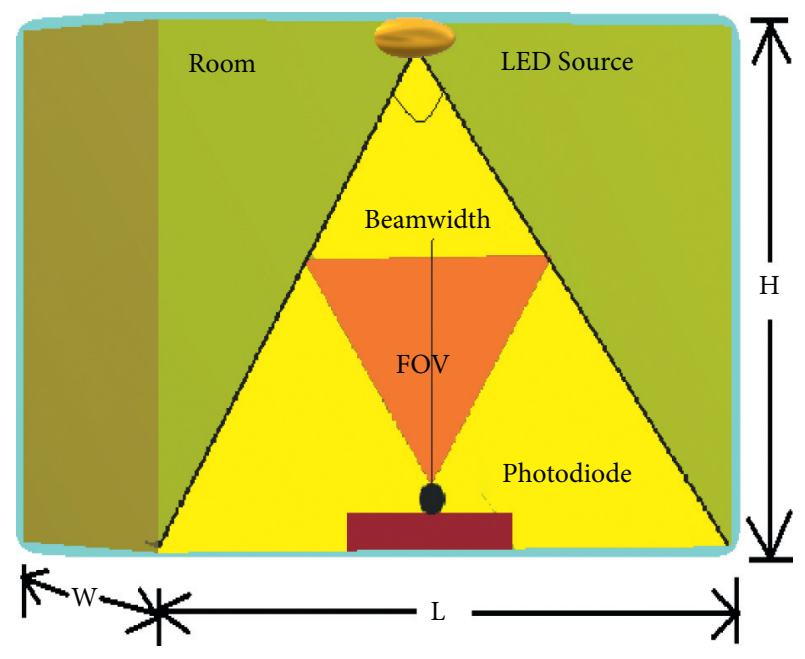

FIGURE 1: System model of LOS link.

one is LED sources completely work under the Lambertian radiation Pattern phenomenon. Practically, it is not a compulsion that LEDs follow LRP, e.g., Phosphor-coated multichip LED does not pursue the Lambertian model for the reproduction of luminous intensity pattern [19].

3.1. Signal Transmission. VLC is relatively a safe technology when it comes to eavesdropping because optical radiations do not permeate through walls which makes them well confined. Switching properties of LEDs in the visible spectrum are considered as the most important constraint that has a rapid capability to be switched on and off because of which it makes it possible to analyze data through impression on the human eye with their radiated power and optical intensity [23, 24]. A human eye can easily observe and detect ultrahigh-speed switching, which is not possible in other technologies. LEDs are considered as an excellent transmission source because of their energy-efficient phenomenon. The Color of LED plays an important role during the setting of the average luminous flux of the source, which is directly proportional to the relative width of the dimming signal [25]. The minimum illumination level for a typical office environment demands illuminance in between 200-1000 lx [26]. Colors that are considered for the OLOS link model during comparative analysis are red $(665 \mathrm{~nm})$, green $(550 \mathrm{~nm})$, blue $(470 \mathrm{~nm})$, and yellow $(600 \mathrm{~nm})$.

Luminance, hue, and saturation are three primary major attributes for the selection of color during conscious sensation. Luminous (often called brightness) is the most preferred one among these attributes, which is based upon luminous and radiant intensities. The second major attribute is the hue, which is related directly to the wavelength. It indicates the separation between color levels, like the distinction between redness, greenness, blueness, etc. The third attribute is saturation chroma, which is used to distinguish the strong colors from faint ones [27]. On the other hand, saturation level shows the freedom from dilution caused by white color, also known as the physical purity of the signal. Therefore, LRP is analyzed here on the performance level of color/wavelength of the source having rotational symmetry.

Mathematical calculations of LRP symmetry rely on Spectral Luminous Efficacy and Spectral Luminous Efficiency. Luminosity factor or Spectral luminous efficacy of radiant flux $\mathbf{K}(\lambda)$ is defined as the quotient of the luminous flux at a certain wavelength by the radiant flux [27].

$$
\mathrm{K}(\lambda)=\frac{\varphi_{\mathrm{V \lambda}}}{\varphi_{\mathrm{e} \lambda}}\left(\text { in } \operatorname{lm} \mathrm{W}^{-1}\right) .
$$

The optimum value of the $\mathbf{K}(\lambda)$ function is $673 \mathrm{~lm} \mathrm{~W}^{-1}$ achieved at a wavelength of about $555 \mathrm{~nm}$ [19]. Related to Spectral Luminous Efficacy, Spectral Luminous Efficiency of radiant flux $\mathbf{V}(\lambda)$ is defined as the ratio of luminous efficacy at a certain wavelength with the value of the maximum luminous efficacy.

$$
\mathbf{V}(\lambda)=\frac{\mathbf{K}(\lambda)}{673} .
$$

Luminous intensity (LI), as discussed earlier, shows the level of brightness represents the overall energy radiated from source and is defined as Luminous Flux per unit solid angle,

$$
I=\frac{d \varphi}{d \Omega}
$$

where $\varphi$ is the luminous flux and $\Omega$ is the spatial/solid angle.

Lambertian distribution pattern for a typical room environment is defined in terms of radiation intensity, that is,

$$
\mathbf{I}(\varnothing)=\mathbf{I}(0) \cos ^{m_{l}}(\varnothing),
$$

where $\varnothing$ is denoting the angle of irradiance relative to the normal axis of the transmitter surface, $\mathbf{I}(0)$ represents the central luminous intensity, and $\mathbf{m}_{\mathbf{l}}$ is the order of radiation lobe in Lambertian emission defined as follows [13]:

$$
\mathbf{m}_{1}=\frac{\ln (2)}{\ln \left(\cos \varnothing_{(1 / 2)}\right)}
$$

where $\Phi_{(1 / 2)}$ is the half-power/semiangle with $\Phi_{(1 / 2)} \in[-\pi / 2, \pi / 2]$ at half illuminance of source provided by the manufacturers of the LED product in their datasheet. Half power angle has a direct influence on the directivity of the transmitter by showing the maximum value of the lobe that contains $50 \%$ of radiant energy in a plane.

The radiation pattern is given by the following:

$$
\mathscr{R} \mathscr{E}\left(\boldsymbol{\varphi}, \mathbf{m}_{1}\right)=\frac{\left(\mathbf{m}_{1}+1\right)}{2 \pi} \cdot \mathbf{P E} \cdot \cos ^{\mathbf{m}_{1}}(\boldsymbol{\varphi}),
$$

where $\mathrm{PE}$ is the transmitting power and $m_{l}$ gives the direction of the source, also known as mode number of radiation lobe.

3.2. Reception. The PIN photodiode is used here as a receptor to collect the light wave. Modeling was performed for luminous intensity distribution pattern at receiving plane in an OLOS path (assumption as neglecting the reflection of walls). The photodiode is used here to convert the optical 
signal as a light wave into an electrical signal which is further enhanced and conditioned by filters. The effective area of receiver or operative signal collection area $\left(\mathbf{A}_{\text {eff }}\right)$ is directly correlated with Field of View (FOV) as follows:

$$
\mathbf{A}_{\text {eff }}(\boldsymbol{\sigma})=\mathbf{A}_{\mathbf{d}} \cdot \cos (\boldsymbol{\sigma})|\boldsymbol{\sigma}|<\text { FOV otherwise } 0,
$$

where $A_{d}$ denotes the physical area of the detector that is considered as small as one of the assumptions and $\sigma$ indicates the direction of the angle between receiver axis at receiving plane and incident light ray as shown in Figure 2. The maximum value of $\sigma$ is directly called FOV of detection of light. The detection capability of the photodiode can be increased by decreasing the FOV value that directly cuts off unwanted reflections through walls and noise at the receiver.

A large area of photodetector causes several problems, such as junction capacitance increases with a reduction in receiver bandwidth. Receiver noise also increases, which will hike the manufacturing cost of the photodetector. Therefore, a nonimaging concentrator is attached as a solution to increase the overall effective area of the receiver. Optical gain of concentrator having a refractive index is considered as follows:

$$
\mathbf{G}(\psi)=\frac{\mathbf{n}^{2}}{\sin ^{2}(\psi)},
$$

where $\psi$ is the Field of View angle of the photodetector.

Radiant flux falling over the receiver also affects the channel gain of the link in which the receiver is located at the distance of $d$ and with an angle of $\phi$ with the OLED. Channel gain calculation for LLIRP requires a receiver connected with a bandpass filter of transmission value $\mathbf{T}_{\mathbf{s}}(\psi)$ and the gain value of nonimaging concentrator [19],

$$
\mathbf{H}=\frac{A_{\text {eff }}\left(m_{1}+1\right)}{2 \pi d^{2}} \cos ^{m_{1}} \varphi T_{s}(\psi) G(\psi) \cos (\psi)
$$

Received power of OLOS link based on illuminance or intensity at a point $(\mathbf{x}, \mathbf{y})$, can be measured with the following:

$$
\begin{aligned}
I_{(x, y)} & =I(0) \frac{\cos ^{m_{1}}(\varnothing)}{d^{2}} \cos \psi \\
P_{r} & =P_{t} \frac{\left(m_{1}+1\right)}{2 \pi d^{2}} \cos ^{m_{1}}(\varnothing) \cdot T_{s}(\psi) \cdot G(\psi) \cdot \cos (\psi) .
\end{aligned}
$$

By using equation (10) in equation (11), we obtain a new factor for LLIRP analysis:

$$
\mathbf{P}_{\mathbf{r}}=\mathbf{P}_{\mathbf{t}} \frac{\left(\mathbf{m}_{1}+1\right)}{2 \pi} \frac{\mathbf{I}_{(\mathbf{x}, \mathbf{y})}}{\mathbf{I}(0)} \cdot \mathbf{T}_{\mathbf{s}}(\psi) \cdot \mathbf{G}(\psi)
$$

Equation (12) illustrates the dependency of received power on the ratio of radiant intensity $(\mathbf{I}(\mathbf{x}, \mathbf{y}))$ in the direction of viewing angle with the luminous intensity pattern (I $(0)$ ) of the LED transmitter. Let us consider a Luminous Intensity Index $\mathbf{S}(\mathbf{x}, \mathbf{y})$ that can be expressed as follows:

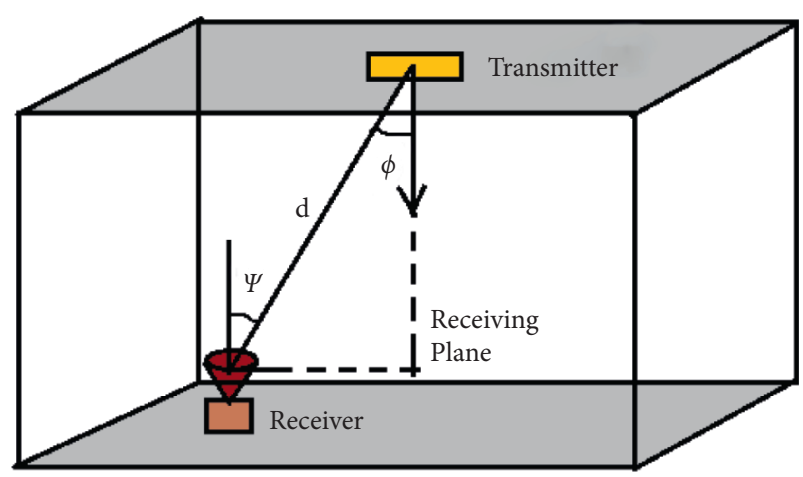

Figure 2: Geometry of OLOS propagation model.

$$
\frac{\mathbf{I}_{(\mathbf{x}, \mathbf{y})}}{\mathbf{I}(0)}=\mathbf{S}(\mathbf{x}, \mathbf{y})
$$

\section{Simulation Results}

Simulation of results on OLOS model performed experimentally for short link communication under an indoor environment with certain room dimensions. In a room, for the analysis of LLIRP, some of the assumptions are considered here as the position of the LED source is located at the top level and in the center of the room while photodiode is situated at ground level, perfectly aligned with the source. Analysis of results completed at different values of the wavelength of light in visible spectrum showing variable colors when detected with human photopic [21] vision. The selection of color process is based on the availability of LED sources in the market. In general, red, green, blue, and yellow LEDs are easily available and very frequently used during most of the research and technical work. Wavelengths severely affect the factor of Spectral Luminous Efficiency of the beam of light, which has been observed during the simulation process while at the same time other parameters are kept constant. Table 1 shows the details of technical specifications being considered simulation process on MATLAB.

Plots indicate the LLIRP of OLED having different colors with the room dimensions. Direction angle relative with transmitter normal axis or half-power angle $(\varnothing)=60^{\circ}$, height between photodetector and transmitter, $h=2.15 \mathrm{~m}$. Field of view $=60^{\circ}$, which is assumed as the half-power angle of the source. The peak value of Luminous intensity is placed at the center of the room for each wavelength but with the variation in brightness level. The wavelength of green light shows the best performance $(4000 \mathrm{~cd})$ under the same conditions when it comes to the illuminance of light, while the wavelength of red $(250 \mathrm{~cd})$ shows a minimum. LLIRP indicates that the performance level in luminous intensity of Green LED is around 16 times more than Red LED.

As shown in Figure 3, the LLIRP of green LED represents the maximum potential in terms of luminous intensity, which is measured in Candela (cd). This plot indicates a range that varies from $2000 \mathrm{~cd}$ to $4000 \mathrm{~cd}$ in particular room dimensions. On the other hand, the yellow LED in Figure 4 shows the next best performance for the same parameters 
TABLe 1: Parameters of modeling LOS link.

\begin{tabular}{lc}
\hline Parameters & Values \\
\hline Room dimensions & $5 \times 5 \times 3 \mathrm{~m}^{3}$ \\
Beam width & $60^{\circ}, 33^{\circ}, 27^{\circ}, 22^{\circ}$ \\
Mode number of radiation lobe, $\mathrm{m}$ & $1,4,6,10$ \\
Field of view $(\psi)$ & $60^{\circ}$ \\
Color of LED & Green, yellow, blue, red \\
Wavelength $(\mathrm{nm})$ & $550,600,470,665$ \\
Spectral luminous efficacy [27] & $0.9950,0.6310,0.0910,0.0610$ \\
Active area of photodiode & $10^{-4} \mathrm{~mm}^{2}$ \\
Refractive index of lens placed at PD & 1.5 \\
Transmitted optical power by LED & $20 \mathrm{~mW}^{2}$ \\
Optical filter gain, $T(\psi)$ & 1 \\
Receiver height $\left(h_{R}\right)$ & $2.15 \mathrm{~m}$ \\
\hline
\end{tabular}

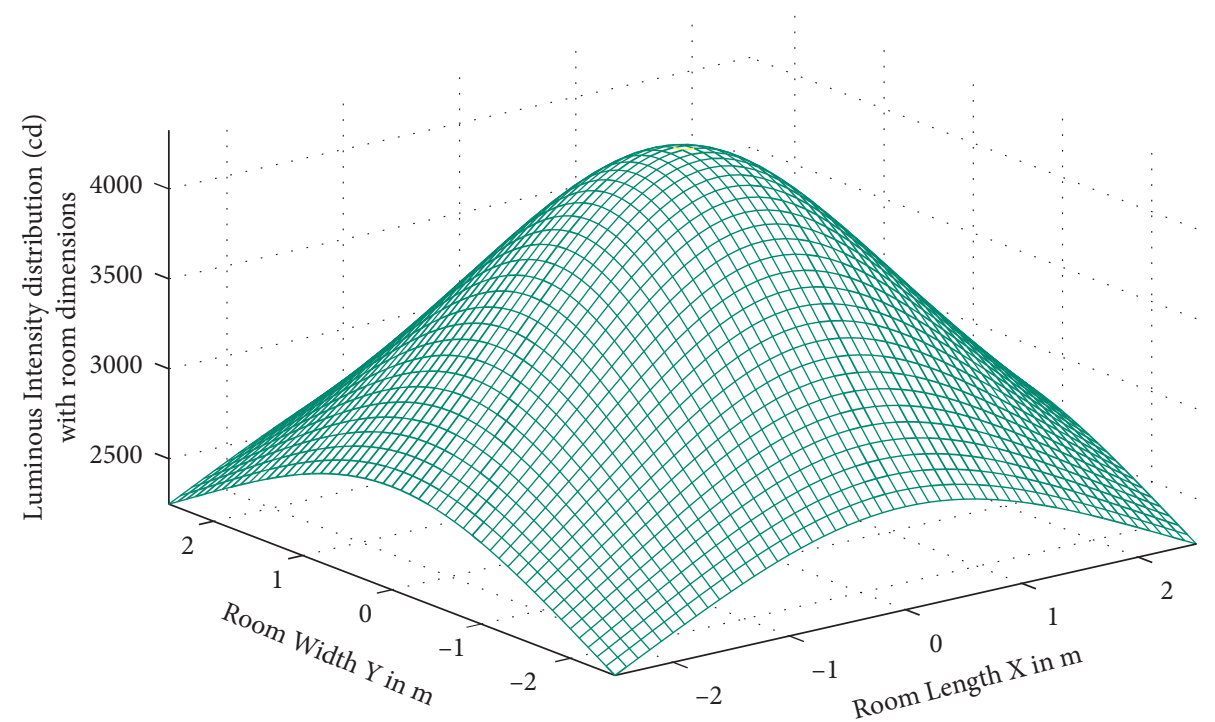

Figure 3: Lambertian radiation pattern for luminous intensity distribution at $550 \mathrm{~nm}$ (green LED).

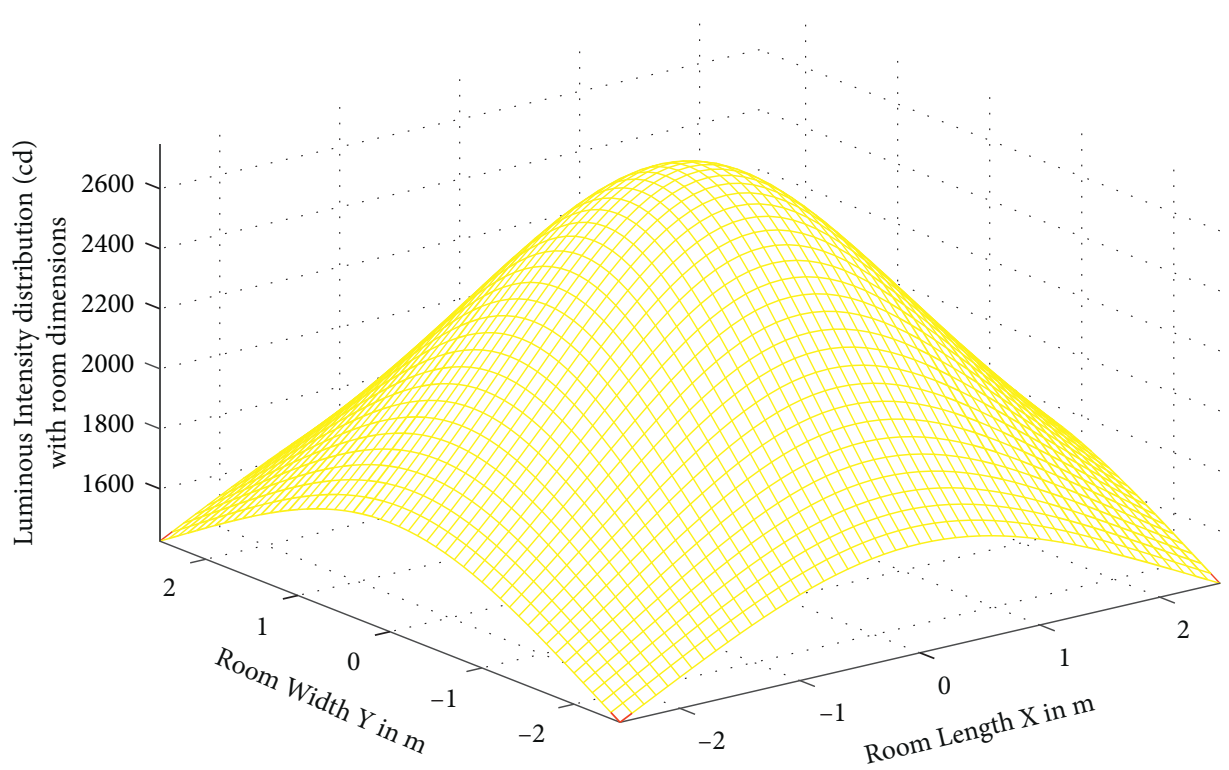

FIGURE 4: Lambertian radiation pattern for luminous intensity distribution at $600 \mathrm{~nm}$ (yellow LED). 
but with different luminous efficacy that is fixed for a certain wavelength, as shown in Table 1. Its luminous intensity varies between $1400 \mathrm{~cd}$ to $2600 \mathrm{~cd}$ for the same dimensions of the room. Blue LED in Figure 5 illustrates a low brightness level as less energy radiates in the range of $200 \mathrm{~cd}$ to $350 \mathrm{~cd}$. Minimum performance of LLIRP if given by red LED radiates lower energy than others shown in Figure 6 that ranges in between $100 \mathrm{~cd}$ and $250 \mathrm{~cd}$.

Figure 7 shows a 2D analysis between LLIRPs of OLED at different wavelengths. Comparative analysis has been presented here that reveals a huge difference in Luminous Intensity of OLEDs that clearly affects the radiation patterns. Peak values of luminous intensity for different cases under the same conditions are shown in the form of a ratio as follows:

$$
\mathrm{G}: \mathrm{Y}: \mathrm{B}: \mathrm{R}=80: 52: 7: 5 \text {. }
$$

Equation (14) indicates the level of difference between LIs of four different cases. The left-hand side of the equation stands for the color of LED, while the right hand signifies the amount of variation in the peak level of LIs. Now, the impact of these performances of Luminous Intensities can be analyzed on other important constraints to increase the credibility of the OLOS link in VLC for more reliable communication.

Based on LLIRP of OLED, analysis of received power distribution has been performed at different wavelengths. Equation (13) shows luminous intensity index, $S(x, y)$, plays a major role in estimating received power at photodetector, which is operated under the impact of various luminous intensity values. In addition, variation in the values of halfpower angle of transmitting OLED have been made, that are set on $\phi=60^{\circ}, 33^{\circ}, 27^{\circ}$, and $22^{\circ}$. Half power angle corresponds to the mode numbers of radiation lobe, which are $m=1,4,6$, and 10 , respectively.

Figure 8 illustrates the outcomes achieved at four different wavelengths as Green, Yellow, Blue, and Red light are the same. Based on the location of the photodetector (in Figure 2) with the source of light, Figure 8 explains that amount of received power is maximum at near about $1 \mathrm{~m}$ of light source, which has been dropped drastically for each case with the increase in distance. General expressions of received power indicate the variation in received power with Luminous Intensity of light, but when the simulation is performed, it shows a different scenario. A new conspiracy is generated, which explains that experimentally color of light does not affect the value of received power when it is operated at the same transmitting power with equivalent mode number of radiation lobe. In other words, $S(x, y)$ does not alter the value of received power if the half power angle of source with its generated power is the same for all LEDs. We obtain the same results as Figure 8, when the LLIRP of the system varies with the wavelength of light due to the complete dependency on the values of $\phi$ and $m$, respectively. For all cases that deals with different colors of OLED, the peak value of received power at the distance of $1 \mathrm{~m}$ as shown in Figure 8, is the same as follows: $0.39 \times 10^{-3}$ at $m=1$, $0.48 \times 10^{-3}$ at $m=4,0.7 \times 10^{-3}$ at $m=6$ and $1.1 \times 10^{-3}$ at $m=10$, respectively.
This work presents the analysis of radiation patterns by using commonly available LEDs. It becomes significant to understand numerous physical properties and characteristics such as LED color, luminous intensity, power gain, transmission/reception power, and peak intensity before developing an efficient interface. The peak intensity of every color (whether it is red or green) is located optimum at $60^{\circ}$ to $70^{\circ}$, whereas the half value of peak intensity shows center intensity. An angle range of $65^{\circ}-75^{\circ}$ is beyond center intensity that is symmetric to the normal axis. Uniform illumination intensity can be obtained from the difference between peak and center intensity onto the target area. Such a study also helps in eliminating the further glare effect via cut-off emitting light whose emitting angle is greater than $80^{\circ}$.

We tried to find out the best possible results by considering the specifications mentioned in Table 1 by using the far-field patterns of LEDs and developing the secondary optics element for multiple propagations. As the imprinted structure of LED can generate the flat far-flied pattern for the center intensity ratio under a superior circumstance. It also delivers the consistent intensity at the $0-40^{\circ}$ distributive angles. In last, the designed secondary optics element can attain the 50\% intensity angle and peak intensity in order to eliminate the glare effect. This work also considers the reflective surface for one reflection in order to avoid the flux loss at the time of interface transmission. VLC transmitters generally utilize LEDs where several LEDs are present in offthe-shelf LED light bulbs. Such light bulbs also involve a driver to maintain the photocurrent that directly affects the Lambertian pattern or luminous intensity. In other words, one can easily manipulate LEDs at a high frequency as transistors control the current arriving at LEDs, conducting communication that is untraceable by human eyes. Normally, VLC works with receivers using PDs. Although, PDs are highly sensitive for human eyes and can handle the waves outside the visible light spectrum like infrared and ultraviolet. Therefore, PD often fails to obtain the data in an outdoor condition exposed to sunlight due to high interference. Some experiments suggest substitutions in order to sort out this challenge. For instance, LEDs have photo sensor characteristics that make them efficient receivers. The LEDs utilization as trans-receiver provides greater flexibility in the applications of VLC models.

LEDs have the property of photocurrent that also make them a good sensor. When LED receives the light, it can produce a small current. This current has magnitude related to the intensity of received light. LEDs can be considered a selective PD, as PD has a wider spectral response. Generally, an LED of a definite color can recognize the signals coming from the same color LED or greater frequencies as they detect a narrower wavelength range. For instance, a blue LED is only capable of recognizing the signals coming from the same color, while a red LED identifies signals from RGB LEDs. We consider multiple LEDs in this work in order to develop an inclusive and comprehensive examination of LED characteristics as sensors, ranging from lesser frequencies (red and yellow) to greater frequencies (blue and green). This work can be further extended to assess the LED 


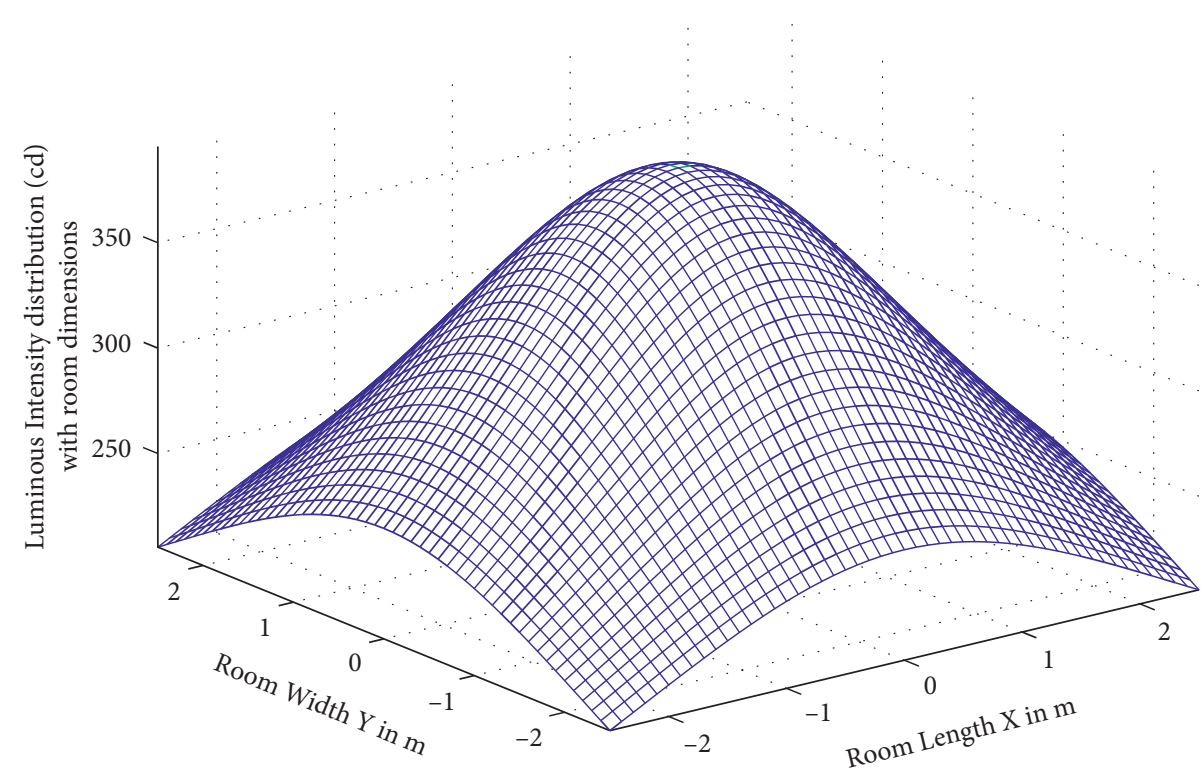

FIGURE 5: Lambertian radiation pattern for luminous intensity distribution at $470 \mathrm{~nm}$ (blue LED).

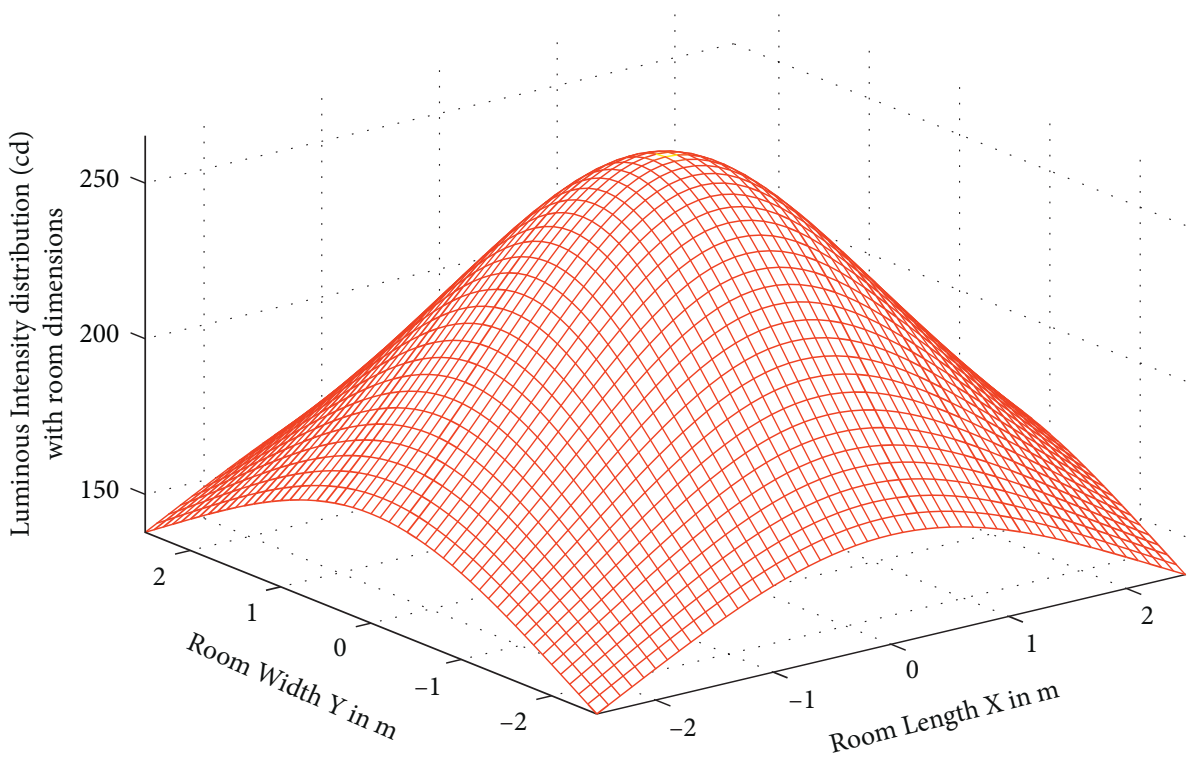

Figure 6: Lambertian radiation pattern for luminous intensity distribution at $665 \mathrm{~nm}$ (red LED).

characteristics by studying several color compositions such as white LED. Further, it helps analyze the materials that can emit radiation, having a mixture of yellow and blue colors. This work considers the experimental studies with the evaluations under practical environment dimensions appropriate for offices, rooms, and labs, etc., that are inclined to forthcoming VLC models because of the existing infrastructure.

\section{Discussion on Work Novelty}

Modeling and simulation of VLC links for OLOS propagation under indoor OWC are highly dependent on key characteristics of the transmitter, receiver, and channel. The objective of conducted work is to present one mathematical model that connects all the variable constraints. The simulation results explain the consequences and dependency between the transmitter and receiver parameters. The various experiments demonstrated in this chapter are discussed as follows:

(i) Color/wavelength is a very important constraint to attain the optimum performance of the link under the same scenario. Comparative results explain that the performance of green color LED in terms of LLIRP is much more effective than other color LEDs. These outcomes help during wavelength selection and support WDM. The impact of wavelength in the visible spectrum has been checked on LLIRP for the VLC link. Results show a clear and 


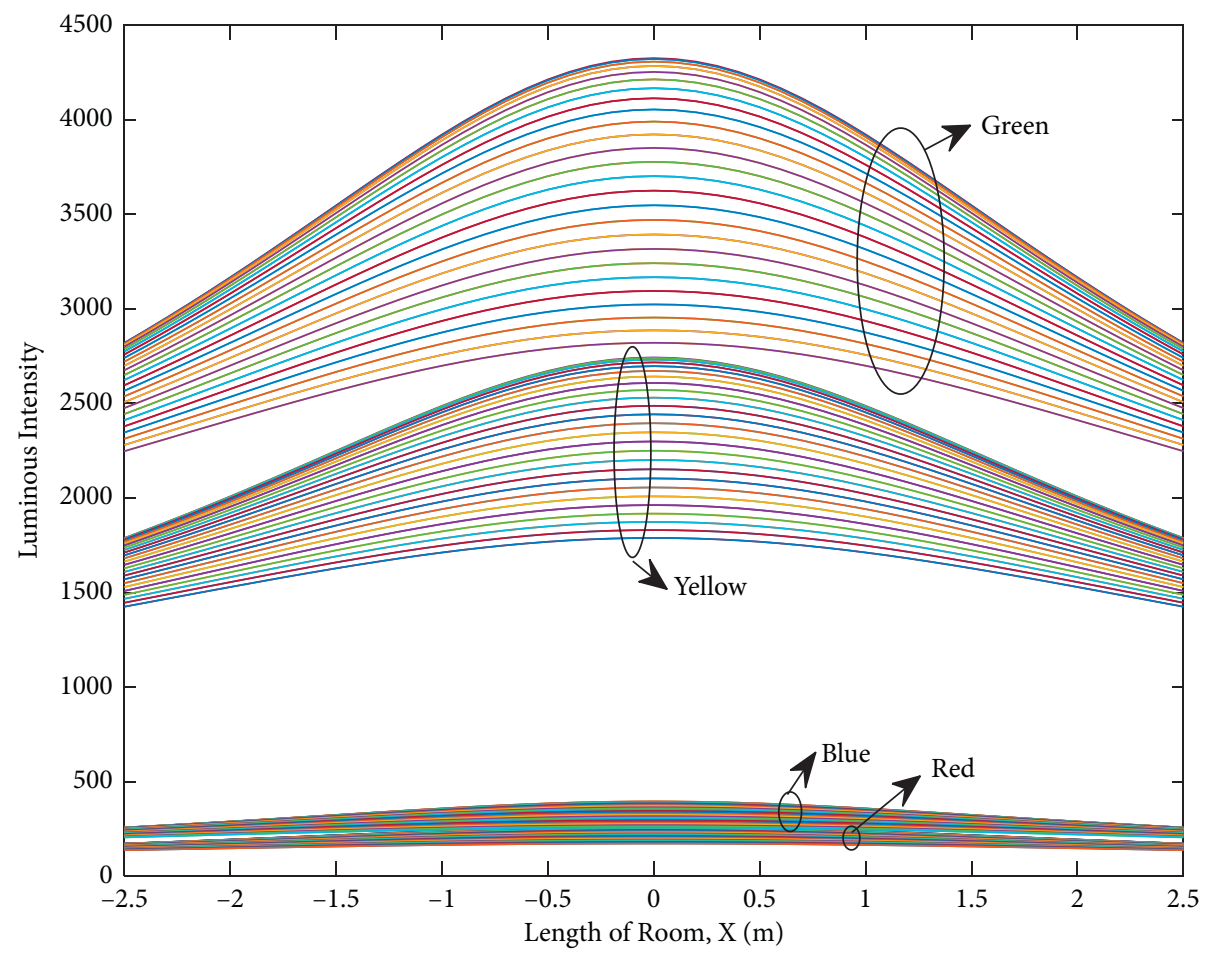

FIgURe 7: Comparative analysis between LLIRP of various wavelengths in 2D plot.

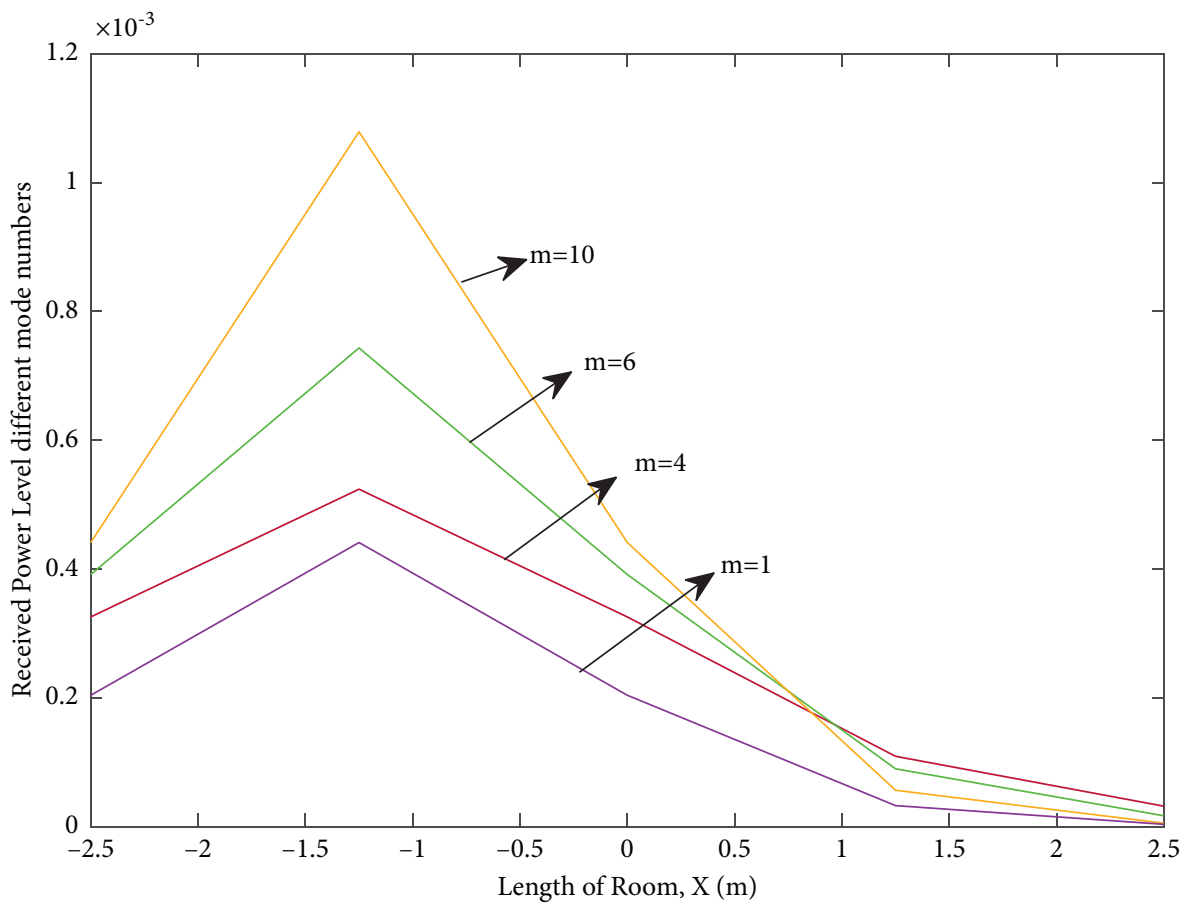

FIgURE 8: Received power distribution of green, yellow, blue, and red LEDs at different mode numbers, $m=1,4,6,10$.

notable variation in the levels of luminous intensity with the room dimensions when the color of light or wavelength varies. Therefore, the selection of light is also an important criterion while creating a reliable OLOS communication link. (ii) Transmitting end depends on two factors: half power angle and the mode number of radiation lobe. Variation in arrival angle represents different mode numbers. Here, the results of four cases have been explained at the HPA of $60^{\circ}, 33^{\circ}, 27^{\circ}$, and $23^{\circ}$. 
Mode number, $m_{1}=1$ at $60^{\circ} \mathrm{HPA}$ represents an ideal Lambertian source that explains the direction where the radiated signal strength reaches the maximum. This work also compares the outcomes generated due to the impact of different mode numbers on the VLC system.

(iii) Receiving section included the FOV and effective area of the photodetector as the main constraints to attain optimum performance.

(iv) Coordinates of transmitter and receiver with rotational symmetry are also important parameters during the investigation of mathematical models.

(v) In addition, the influence of luminous intensity has been noted on the further impact of brightness and optical received power. It displays that the color of light does not influence the received power of the photodetector. The behavior of spectral luminous efficacy, radiation flux, and luminous intensity of the source has been checked with the receiving section, including a nonimaging concentrator and a bandpass filter. $S(x, y)$ found constant for all colors, whether it is green, yellow, blue, or red; each wavelength gives the same plot in case of received power. The only change is detected because of the variations in values of the number of modes caused by the half-power angle of transmitting source LED.

\section{Conclusion}

In this investigation, a new model of OLOS for indoor VLC systems has been proposed. It is widely applicable for the further analysis of LLIRP. Several parameters can affect the whole OLOS system with a minute change in values. It can include the coordinates of the transmitting source with the location of the photodetector or the half-power angle of OLED with their respective mode number of radiation lobe. This extension of work analyzes a new model that is created by changing the luminous intensity of light. The wavelength impact of light in the visible spectrum has been checked on the Lambertian radiation pattern of the lobe in a VLC link. Results show a clear variation in Luminous intensity levels with the room dimensions when wavelength varies. Therefore, the selection of light is also an important criterion while creating a reliable OLOS communication link. In addition, the luminous intensity factor is used to check the further impact of brightness on the factor of received power. It shows that the color of light does not influence the factor of the received power of the photodetector. The behavior of radiation flux, Spectral luminous Efficacy, and luminous intensity of source have been checked with the receiver connected with a nonimaging concentrator and a bandpass filter. $S(x, y)$ found constant for all colors, whether it is green, yellow, blue, or red. Each wavelength gives the same plot in case of received power. The only change is observed due to changes in values of half-power angle and the number of modes of the transmitter.

Future work will be executed on other parameters that are linked with LLIRP. Parameters like bit error rate (BER) or signal to noise ratio (SNR) will be investigated and further efforts will be made to find out a relation between the impacts of luminous intensity or OLED radiation patterns with these factors.

\section{Data Availability}

The authors confirm that all relevant data are included in the article and its information files. Additionally, the derived data supporting the findings of this study are available from the corresponding author upon request.

\section{Conflicts of Interest}

The authors declare that they have no conflicts of interest.

\section{Acknowledgments}

This work was supported by Universidad Autonoma de Zacatecas, Mexico and CONACyT, Mexico.

\section{References}

[1] D. K. Borah, A. C. Boucouvalas, C. C. Davis, S. Hranilovic, and K. Yiannopoulos, "A review of communication-oriented optical wireless systems," EURASIP Journal on Wireless Communications and Networking, vol. 1, pp. 1-28, 2012.

[2] M. A. Khalighi and M. Uysal, "Survey on free space optical communication: a communication theory perspective," IEEE Communications Surveys \& Tutorials, vol. 16, no. 4, pp. 2231-2258, 2014.

[3] D. Tsonev, S. Videv, and H. Haas, "Light fidelity (Li-Fi): towards all-optical networking," in Broadband Access Communication Technologies VIII, International Society for Optics and Photonics, Bellingham, WA, USA, 2014.

[4] R. Sharma, A. Charan Kumari, M. Aggarwal, and S. Ahuja, "Optimal LED deployment for mobile indoor visible light communication system: performance analysis," AEU-International Journal of Electronics and Communications, vol. 83, pp. 427-432, 2018.

[5] R. X. G. Ferreira, E. Xie, J. J. D. McKendry et al., "High bandwidth GaN-based micro-LEDs for multi-Gb/s visible light communications," IEEE Photonics Technology Letters, vol. 28, no. 19, pp. 2023-2026, 2016.

[6] H. Hoa Le Minh, D. O’Brien, G. Faulkner et al., “100-Mb/s NRZ visible light communications using a postequalized white LED," IEEE Photonics Technology Letters, vol. 21, no. 15, pp. 1063-1065, 2009.

[7] H. Elgala, R. Mesleh, H. Haas, and B. Pricope, "OFDM visible light wireless communication based on white LEDs," in Proceedings of the 2007 IEEE 65th Vehicular Technology Conference-VTC2007-Spring, pp. 2185-2189, IEEE, Dublin, Ireland, April 2007.

[8] Y. Wang and H. Haas, "Dynamic load balancing with handover in hybrid $\mathrm{Li}-\mathrm{Fi}$ and Wi-Fi networks," Journal of Lightwave Technology, vol. 33, no. 22, pp. 4671-4682, 2015.

[9] C.-L. Tsai and Z.-F. Xu, "Line-of-sight visible light communications with InGaN-based resonant cavity LEDs," IEEE Photonics Technology Letters, vol. 25, no. 18, pp. 1793-1796, 2013.

[10] M. C. Al Naboulsi, H. Sizun, and F. de Fornel, "Wavelength selection for the free space optical telecommunication technology," in Reliability of Optical Fiber Components, Devices, 
Systems, and Networks II, pp. 168-179, International Society for Optics and Photonics, Bellingham, WA, USA, 2004.

[11] O. Bouchet, M. El Tabach, M. Wolf et al., "Hybrid wireless optics (HWO): building the next-generation home network," in Proceedings of the 2008 6th International Symposium on Communication Systems, Networks and Digital Signal Processing, pp. 283-287, IEEE, Graz, Austria, July 2008.

[12] I. Moreno and C.-C. Sun, "Modeling the radiation pattern of LEDs," Optics Express, vol. 16, no. 3, pp. 1808-1819, 2008.

[13] G. Stepniak, M. Kowalczyk, L. Maksymiuk, and J. Siuzdak, "Transmission beyond $100 \mathrm{Mbit} / \mathrm{s}$ using LED both as a transmitter and receiver," IEEE Photonics Technology Letters, vol. 27, no. 19, pp. 2067-2070, 2015.

[14] P. Dietz, W. Yerazunis, and D. Leigh, "Very low-cost sensing and communication using bidirectional LEDs," in Proceedings of the International Conference on Ubiquitous Computing, pp. 175-191, Springer, Berlin, Heidelberg, October 2003.

[15] D. Giustiniano, N. O. Tippenhauer, and S. Mangold, "Lowcomplexity visible light networking with LED-to-LED communication," in Proceedings of the 2012 IFIP Wireless Days, pp. 1-8, Dublin, Ireland, November 2012.

[16] S. Li, B. Huang, and Z. Xu, "Experimental MIMO VLC systems using tricolor LED transmitters and receivers," in Proceedings of the 2017 IEEE Globecom Workshops (GC Wkshps), pp. 1-6, Singapore, December 2017.

[17] Z. Ahmad, S. Rajbhandari, O. Salih, and R. Green, "Demonstration of a multi-hop underwater visible light communication system," in Proceedings of the 2017 19th International Conference on Transparent Optical Networks (ICTON), pp. 1-4, Girona, Spain, July 2017.

[18] M. Heydariaan, S. Yin, O. Gnawali, D. Puccinelli, and D. Giustiniano, "Embedded visible light communication: link measurements and interpretation," in Proceedings of the 2016 International Conference on Embedded Wireless Systems and Networks (EWSN 2016), Graz, Austria, February 2016.

[19] M. V. Bhalerao, M. Sumathi, and S. S. Sonavane, "Line of sight model for visible light communication using Lambertian radiation pattern of LED," International Journal of Communication Systems, vol. 30, no. 11, p. e3250, 2017.

[20] Y. Wang, M. Chen, J.-Y. Wang et al., "Impact of LED transmitters' radiation pattern on received power distribution in a generalized indoor VLC system," Optics Express, vol. 25, no. 19, pp. 22805-22819, 2017.

[21] W.-T. Chien, C.-C. Sun, and I. Moreno, "Precise optical model of multi-chip white LEDs," Optics Express, vol. 15, no. 12, pp. 7572-7577, 2007.

[22] I. Moreno, "Spatial distribution of LED radiation," in Proceedings of the International Optical Design Conference 2006, International Society for Optics and Photonics, Vancouver, Canada, July 2006.

[23] D. O’Brien, L. Zeng, H. Le-Minh et al., Visible Light Communication, Short-Range Wireless Communications: Emerging Technologies and Applications, Wiley Publishing, Hoboken, NJ, USA, 2009.

[24] H. Le Minh, D. O’Brien, G. Faulkner et al., "High-speed visible light communications using multiple-resonant equalization," IEEE Photonics Technology Letters, vol. 20, no. 14, pp. 1243-1245, 2008.

[25] S. Rajbhandari, Optical Wireless Communications: System and Channel Modelling with MATLAB ${ }^{\circledR}$, CRC Press, Boca Raton, FL, USA, 2019.

[26] F. R. Gfeller and U. Bapst, "Wireless in-house data communication via diffuse infrared radiation," Proceedings of the IEEE, vol. 67, no. 11, pp. 1474-1486, 1979.

[27] R. W. Waynant and M. N. Ediger, Electro-Optics Handbook, McGraw-Hill Education, New York, NY, USA, 2000. 